\title{
Factors influencing the yield of detections in CoRoT
}

\author{
J. Cabrera ${ }^{1}$ and the CEST Team \\ ${ }^{1}$ Institut für Planetenforschung, Deutsches Zentrum für Luft- und Raumfahrt, \\ Rutherfordstr.212489 Berlin [juan.cabrera@dlr.de]
}

\begin{abstract}
The CoRoT mission has observed more than 100000 stars since its launch in December 2006 and has found so far 13 planets and 2 brown dwarves. We are studying the factors affecting the yield of detections in the different CoRoT runs.
\end{abstract}

\section{The yield of detections in CoRoT}

Since its launch and until August 2010 CoRoT has observed more than 100000 stars in 15 different fields. CoRoT points towards two defined regions of the sky: the center direction is located towards the constellation of Aquila (galactic longitude $40^{\circ}$ ) and the anti-center direction towards the constellation Monoceros (galactic longitude $210^{\circ}$ ). According to the length of the observations there are three categories of runs: the short runs (around 25 days long), the medium runs (from 60 to 90 days long) and the long runs (up to 145 days long).

As of August 2010, 13 planets and 2 brown dwarves have been published: 7 planets towards the anti-center direction and 6 planets and 2 brown dwarves towards the center direction. In principle, one would expect to find more planets in the anti-center direction, as the characterization of the CoRoT fields prior to the launch expected a stellar population more favorable for the planet search in this region (Deleuil et al. 2009). On the other hand, 11 planets and 1 brown dwarf were found in long runs, 2 in the IRa01 medium run and 1 brown dwarf in a short run. Indeed, one would expect more detections in a longer run, not only because one integrates more signal and therefore increases the SNR, but also because the photometric follow-up depends on the ephemeris of the planetary candidates, which are better constrained in the long runs (Deeg et al. 2009). Finally, all planets and brown dwarves have been found around main sequence stars. The discovery of transiting planets around giant like stars (class III) is challenging because these stars have big radii (10 solar radii is a typical value for Red Clump Giants). A Jupiter-sized planet around one of these giants would have long period (more than 16 days if orbiting its host star at 4 stellar radii) and a depth below $0.01 \%$.

It is difficult to do a statistical analysis of the planetary yield of CoRoT with a sample of 13 planets. However, from the detection point of view, the technical requirements to detect in series of data a periodic signal of a given depth do not distinguish between detecting planets and eclipsing binaries. It is true that a Jupiterlike planet would make a transit $1 \%$ deep around a solar-like star whereas, for example, an eclipsing binary where both stars have equal sizes would show $50 \%$ deep eclipses

This is an Open Access article distributed under the terms of the Creative Commons Attribution-Noncommercial License 3.0, which permits unrestricted use, distribution, and reproduction in any noncommercial medium, provided the original work is properly cited. 


\section{EPJ Web of Conferences}

(with a much higher SNR for the same noise level). But many grazing eclipsing binaries have eclipses below the $1 \%$ depth planetary threshold and, on the other hand, Jupitersized planets around late $\mathrm{M}$ stars will show transits much deeper than $1 \%$. Moreover, if we focus just in the photometric detections, we get rid of any possible follow-up observational bias: the telescope time being finite, it is not possible to follow-up every planetary candidate until its nature is fully elucidated.

To perform a statistical study of the yield of detections of CoRoT we will focus on the detection of both planetary candidates (around 40 per run) and eclipsing binaries (around 200 per run) to have a bigger statistical sample. Because of the reasons commented above, from the detection point of view planets and eclipsing binaries are found by CoRoT among the same stellar population of main-sequence stars. ${ }^{1}$

In our preliminary study, we have found that the knowledge of the number of dwarf stars in a run, which is known thanks to the pre-launch characterization of the CoRoT fields, is not enough to account for the yield of detections; independently of the length of the run.

The CoRoT fields are located close to the Galactic plane, where the interstellar extinction is inhomogeneous and significant (Schlegel et al. 1998). We have found a correlation between the interstellar extinction of a field (estimated from the J vs. J-K CMD diagrams, see Indebetouw et al. 2005) and the number of detections in the run, which we are currently investigating.

\section{References}

Deeg et al. 2009 A\&A, 480, 563

Deleuil et al. 2009 A\&A, 138, 649

Indebetouw et al. 2005 ApJ, 619, 931

Schlegel et al. 1998 A\&A, 500, 525

\footnotetext{
${ }^{1}$ Giant eclipsing binaries have been found, but they are rare cases, not statistically significant.
} 